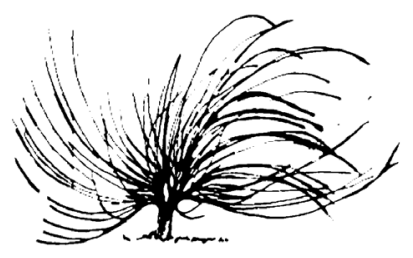

\title{
Perspectiva moral de las emociones en los contextos de educación formal
}

Felipe Nicolás Mujica Johnson ${ }^{1}$

Universidad Politécnica de Madrid

España

fmujica@live.cl

Nelly del Carmen Orellana Arduiz ${ }^{2}$

Universidad de Playa Ancha de Ciencias de la Educación

Chile

norellan@upla.cl

Juan Carlos Luis-Pascual

Universidad de Alcalá

España

juan.luis@uah.es

\begin{abstract}
Resumen
El presente ensayo responde a un análisis crítico de la educación emocional. Considera que las emociones en la educación formal se están abordando desde una perspectiva terapéutica, fundamentada en la tradición hedonista de la psicología, lo cual no corresponde a un conocimiento pedagógico, más aún cuando no se consideran los fundamentos filosóficos de la educación.
\end{abstract}

Recibido: 5 de setiembre de 2018. Aprobado: 10 de abril de 2019.

http://dx.doi.org/10.15359/rep.14-1.4

1 Magíster en Ciencias de la Actividad Física y del Deporte, Universidad de Playa Ancha de Ciencias de la Educación, Chile. Doctorando en Ciencias de la Actividad Física y del Deporte, Universidad Politécnica de Madrid, España.

2 Máster en Educación Física, Escuela Superior de Deporte, Alemania. Doctora en Filosofía y Educación, Universidad Nacional a distancia, España. Doctora en Filosofía, Leibnis Universität, Alemania.

3 Máster en Fundamentos Psicológicos de la Educación, Universidad Autónoma de Madrid, España. Máster en Docencia Universitaria, Universidad de Alcalá, España. Doctor en Filosofía y Ciencias de la Educación, Universidad Complutense de Madrid, España. 
Tomando en cuenta esa crítica, se genera este ensayo que tiene por finalidad analizar el valor moral de las emociones. Se identifica que una misma emoción puede tener un valor positivo o negativo en relación con el significado de la meta educativa. Se concluye que no es posible estandarizar el valor pedagógico-moral de las emociones, ya que responden a un análisis cualitativo de la situación pedagógica.

Palabras clave: Educación moral, afectividad, bienestar social, bienestar del estudiantado, desarrollo emocional.

\begin{abstract}
This essay responds to a critical analysis of emotional education, considering that emotions in education are being addressed from a therapeutic perspective based on the hedonistic tradition of psychology, which does not correspond to a pedagogical knowledge, especially when the philosophical foundations of education are not considered. Taking into account this criticism, this essay is generated which aims to analyze the moral value of emotions. It is identified that the same emotion can have a positive or negative value in relation to the meaning of the educational goal. It is concluded that it is not possible to standardize the pedagogical-moral value of emotions since it responds to a qualitative analysis of the pedagogical situation.
\end{abstract}

Keywords: moral education, affectivity, social welfare, student welfare, emotional development.

\title{
Introducción
}

La educación formal es el espacio institucional más importante para construir la cultura en la sociedad occidental, pero desarrollar las finalidades pedagógicas se torna un tema muy complejo, ya que no existe una sola interpretación del mundo, de modo que ante un concepto que debe ser transferido al contexto educativo, surgen múltiples interpretaciones de cómo se debería aplicar. Uno de esos conceptos es el bienestar, el cual se encuentra proclamado en la declaración de los 
derechos humanos, que es considerada uno de los repertorios morales más importantes (Montero, 2016).

Incluir el bienestar en la educación no ha sido una tarea fácil, dada la amplitud de su concepto, que ha generado diversas interpretaciones en torno a este valor fundamental, por lo que, hasta la actualidad, es un concepto que ha sido delimitado desde diferentes disciplinas, pues la calidad de vida de las personas es un tema relevante en la actualidad para las naciones, que lo abordan desde las diferentes disciplinas académicas, desde el ámbito médico hasta el político (Vázquez, 2009). En la última década, con el auge de la psicología positiva, ha ganado terreno en el ámbito educativo. No obstante, pensar el bienestar humano en la educación resulta algo complicado, por la complejidad y abstracción del concepto, cuya discusión ha suscitado tradicionalmente grandes dificultades interpretativas (Aguado et al., 2012).

En este ensayo, el bienestar se abordará desde un enfoque moral, específicamente su corriente subjetiva, la cual se enmarca en una perspectiva hedonista, que encuentra sus raíces en la filosofía griega, uno de cuyos representantes es Epicuro, creador de una de las primeras escuelas que interpreta el placer como un objeto de estudio en relación con la vida misma, quien declara que los placeres provenientes del pensamiento, de la mente y del alma tienen un privilegio sobre los placeres provenientes del cuerpo humano. Agrega que tanto el alma como la carne son existencias materiales, es decir, corpóreas (Uribe et al., 2017).

Desde este enfoque y una vez establecido el vocabulario emocional que manejamos (Bisquerra y Filella, 2018), el bienestar subjetivo le otorga gran importancia a la percepción de las emociones, valoradas como positivas según el placer (agrado) que producen o negativas, de acuerdo con el dolor (desagrado) que generan en los sujetos. De este modo, este tipo de bienestar se compone de aspectos afectivos, pero también incluye un elemento cognitivo (García, 2002). Por lo tanto, el bienestar subjetivo dependerá de tres factores: la presencia relativa de afecto positivo, la ausencia de afecto negativo y la satisfacción con la vida (Myers y Diener, 1995; Vásquez et al., 2009). Es un modelo tridimensional comprobado en forma empírica en estudiantes con edades comprendidas entre los 12 y los 23 años (Rodríguez-Fernández y Goñi-Grandmontagne, 2011).

Por lo tanto, el argumento que manifiesta esta teoría es que las experiencias placenteras, en las que se experimentan emociones positivas, 
tienen una relación directa con una buena valoración de la vitalidad, de modo que "las personas con un mayor bienestar subjetivo hacen una valoración más positiva de sus circunstancias y eventos vitales; mientras que las "infelices" evalúan la mayor parte de estos acontecimientos como perjudiciales" (Zubieta et al., 2012, p. 67). En consecuencia, el valor de las emociones en esta teoría se encuentra definido a priori y en forma estable. Entiende las positivas como aquellas que favorecen el bienestar y las negativas en un sentido contrario, pues lo disminuyen (Redorta et al., 2006).

Diferentes estudios del ámbito educativo han elaborado propuestas con el fin de incorporar esta teoría en la práctica educativa. En España surge la propuesta con el nombre de "educación emocional" (Bisquerra, 2005; Bisquerra et al., 2016; Bisquerra y Filella, 2003; Bisquerra y Filella, 2018; Bisquerra y Hernández, 2017), una de las más reconocidas en la formación del profesorado y en la práctica escolar, proyectada también a la educación no formal. En esta propuesta se reconocen planteamientos muy acordes a la necesidad actual; sin embargo, se identifica que transferir, en forma estable y a priori, el valor de las emociones al contexto pedagógico genera un vacío moral que no puede ser ignorado, lo cual se transforma en el tema central de este ensayo.

La educación emocional se está convirtiendo en una urgencia pedagógica (Valdés y Gutiérrez, 2018), pero es menester incorporar la perspectiva moral del bienestar, lo cual ha sido uno de los aspectos que ha servido de crítica a este modelo, por lo cual se sugiere "trabajar para incluir los avances en la educación emocional dentro de un marco educativamente más amplio y poderoso que es la educación ética, en el que mantendría su indispensable carácter instrumental" (Marina, 2005, p. 40). En la misma línea argumentativa, se ha señalado que este tipo de modelos, sustentados en la inteligencia emocional y la psicología positiva, promueven una psicologización de la educación, lo cual sería un error, ya que el conocimiento pedagógico no debería estar al servicio de una perspectiva terapéutica, sino más bien al de un fundamento ético de la educación (Prieto, 2018).

Además, se ha llegado a señalar que solo una perspectiva eudaemónica del bienestar debiese ser considerada como objeto de educación, lo cual no es una opinión compartida por el equipo autor de este ensayo, pues el bienestar subjetivo tiene mucho que aportar a la educación, al igual que las emociones tienen una gran relevancia como contenido 
educativo (López, 2016; Maturana, 2001; Toro, 2017). Por lo tanto, la perspectiva hedonista del bienestar no tendría que ser descartada en su totalidad del escenario pedagógico, sino que ser aplicada en el marco ético que constituye las finalidades de la educación.

En la actualidad, la educación formal en occidente se ha consolidado desde una perspectiva constructivista, la cual emerge para reivindicar la complejidad del acto educativo, entendiendo que no se puede mantener una reducción lineal de causa y efecto para orientar el aprendizaje. Esta perspectiva "entiende que el proceso de enseñanza y aprendizaje se vertebra a partir de las relaciones conjuntas entre el profesor y el alumno alrededor del contenido" (López-Ros et al., 2014, p. 34). Con respecto al rol del alumnado en esta visión epistemológica, se comprende que "el alumno no sólo posee unos conocimientos previos sobre muchos de los conceptos básicos de la ciencia escolar, sino que éstos juegan un papel de primera magnitud en la adquisición de nuevos conocimientos" (Perales, 2000, p. 34). Para representar la concepción constructivista del aprendizaje, se afirma que su principal idea "es que el sujeto 'construye' el conocimiento mediante la interacción que sostiene con el medio social y físico" (Sarramona, 2008, p. 249).

Tomando en cuenta aquellos postulados, las emociones también han sido estudiadas desde la perspectiva sociocultural en el campo de la educación (Rebollo et al., 2006; Holodynski y Friedlmeier, 2006), por lo tanto se les otorga una función psicosocial, pues servirían para la integración de las personas en los grupos sociales y para la construcción social de la identidad (Guedes y Álvaro, 2010). En este sentido, también se debe destacar el componente cultural de las emociones, teniendo presente que las personas participan en múltiples contextos formales o informales de la vida social, donde se interactúa con diversos significados y valores rígidos o flexibles en su transformación o reconstrucción social, implícitos en las normativas de cada institución (instituciones educativas, instituciones religiosas, instituciones deportivas, reuniones de amistad, transporte público, entre otros), en las costumbres sociales aceptadas o reprimidas, por consiguiente, en un contextualizado espacio de convivir que contribuye a formar la identidad de sus participantes (Rebollo y Hornillo, 2010). Por esa razón, las emociones han sido descritas como "fenómenos de corta duración, relacionados con sentimientos, estimulación, intención y expresión, que nos ayudan a adaptarnos a las oportunidades y retos que afrontamos durante los sucesos significativos de la vida" (Reeve, 2010, p. 7). 
Con respecto a la constitución de la emoción, esta incorpora los factores biológico, psicológico y sociocultural, descritos por Reeve (2010) en los siguientes cuatro componentes: a) el sentimental o cognitivo-subjetivo: experiencia subjetiva, concienciación fenomenológica y cognición; b) el de estimulación corporal o biológico: activación fisiológica, preparación corporal para la acción y respuestas motoras; c) el sentido de intención: estado motivacional dirigido a metas y aspecto funcional; y d) el social-expresivo o sociocultural: comunicación social, expresión facial y expresión vocal.De esta forma, en el abordaje que se realiza a la emoción en el presente ensayo, se es consecuente con la idea de que las emociones del ser humano son determinadas por "factores biológicos y culturales, como las expresiones faciales, el vocabulario emocional de cada una de las culturas del mundo o, todavía de un modo incompleto, las huellas químicas en nuestro cerebro" (Alonso, 2017, p. 19). Por lo tanto, cuando se habla de cultura, se debe incorporar la perspectiva moral que se encuentra vigente en las naciones occidentales.

Con el fin de aportar a la educación emocional, el objetivo de este ensayo es argumentar la necesidad de considerar un valor pedagógico de las emociones en las tareas educativas, el cual dependería en gran parte del valor moral que se puede atribuir a su sentido de intención.

\section{El bienestar y las emociones en la educación}

El bienestar es reconocido a nivel universal como un derecho social (estar bien, estar a gusto, alcanzar una plenitud y una satisfacción personal), el cual sería aplicable a todos los seres humanos, independiente de cualquier distinción, en virtud de que todas las personas son iguales por naturaleza (Ruiz, 2007); no obstante, la ambigüedad en torno a algunos de los postulados de los derechos humanos genera dudas en cuanto a la efectividad práctica del pensamiento moral (Platts, 2010), como es el caso del bienestar, que, por ser un concepto de tanta amplitud, ha sido abordado desde una corriente subjetiva y objetiva.

El rol asumido por los Estados ante este derecho se ha asociado a la corriente objetiva del bienestar, que hace referencia al nivel de vida de las personas (estado de bienestar), y considera, entre sus variables, el nivel de subsistencia, los bienes materiales no imprescindibles, el respeto, la libertad, las relaciones personales y la cultura. Considerar la corriente subjetiva es difícil, entendiendo que la felicidad, desde esta 
perspectiva, se encuentra asociada a los deseos particulares, los cuales pueden estar motivados por deseos de una variada índole, incluidos motivos inmorales, por lo que si el Estado se basara en esta corriente, se podrían cometer muchos errores. Por lo tanto, se ha argumentado que considerar "el bienestar como la satisfacción del nivel de vida tal y como lo propone la corriente objetiva, tiene la ventaja de no hacer depender el bienestar de una persona, de elementos puramente subjetivos o emocionales" (Briceño y Guillezeau, 2012, p. 32).

Este argumento explica el peligro de que un Estado considere solamente aspectos subjetivos del bienestar, sin embargo, producto de un enorme interés social por el bienestar humano, que no necesariamente se basa en la corriente objetiva, los gobiernos de muchos países han comenzado a incorporar iniciativas en sus agendas políticas que exploran estrategias alternativas o complementarias al producto interior bruto (PIB) al momento de evaluar el bienestar humano y el progreso social de las naciones (Aguado et al., 2012). Una de las alternativas que se propone desde el ámbito psicológico, es la evaluación del bienestar subjetivo (Galí, 2018), también como un complemento de la corriente objetiva en la evaluación del bienestar, con el fin de establecer nuevos indicadores que vayan más allá de lo material y puedan contribuir a la calidad de vida de las personas (Unanue et al., 2017), con ello se trasciende el enfoque dominante de occidente orientado hacia un estilo de vida mercantilizado y deshumanizado (Aguado et al., 2012).

En el terreno de la educación, el bienestar subjetivo podría plantearse como una meta educativa, la cual se tendría que enmarcar en las condiciones éticas de la sociedad o incluso rasgos culturales, en los que se identifican los aspectos positivos y fortalezas (Soriano y Cala, 2018). Ante esta posibilidad, es importante señalar que las emociones en el contexto educativo no pueden mantener el valor otorgado por la teoría del bienestar subjetivo, ya que lo positivo y negativo no estaría únicamente determinado por lo que siente el sujeto, sino por normas objetivas fundadas en la moral. Un ejemplo, de lo anterior, es que la alegría, emoción positiva para el bienestar subjetivo, en el contexto pedagógico puede ser valorada como negativa, debido a que si un estudiante la percibe a causa de insultar a otra persona, con quien anteriormente había protagonizado un conflicto, se convierte en una emoción que refuerza una conducta incorrecta. El valor de las emociones va a variar según el objeto con el que es asociado, como se indica desde un punto de vista 
antropológico: las emociones tienen un significado cultural, político, ético y moral, y se pueden analizar desde un nivel intrasubjetivo, intersubjetivo y transubjetivo (Calderón, 2014).

Producto de la ignorancia sobre el rol de las emociones en la vida personal y social, el ser humano vive sus argumentos racionales sin hacer referencia a las emociones en que se fundan, porque no se sabe que ellos y todas nuestras acciones tienen un fundamento emocional, y se cree que tal condición sería una limitación a nuestro ser racional (Maturana, 2001).

En la actualidad, a medida que se comprende más el funcionamiento del cerebro humano, aumentan los argumentos para señalar que la emoción y la razón se encuentran integradas y formarían una unidad (Cabezas, 2013; Damasio, 2011; Mora, 2017). Desde esta perspectiva integrada, los estados emocionales son descritos como una toma de conciencia de esa valoración que realiza la mente en función del estado con el que enfrenta las diferentes circunstancias, para, de esta forma, adaptar o mantener la conducta para obtener los resultados esperados (Zaccagnini, 2008). En efecto, las emociones experimentadas en las diferentes situaciones vitales, serían suscitadas desde el plano mental, como respuestas a las valoraciones cognitivas (Lazarus, 2000).

En cuanto a los factores que influyen aquellas valoraciones, han sido destacadas las creencias, que pueden ser originadas en el plano racional o irracional, y fundadas en elementos reales (hechos concretos) o irreales (imaginarios) experimentados en el cotidiano vivir, en conjunto con la integración de nuevos conocimientos y percepciones que provienen del diálogo familiar o con amistades; de los medios masivos de comunicación y de la participación en instituciones educativas, religiosas, deportivas o artístico-culturales, en donde la persona desde su infancia aprende a verse como la ven o a valorarse como la valoran (Salmurri, 2015). Por eso, una educación crítica de la emociones tendría que considerarlas como una manifestación social, cultural e histórica, que caracteriza la compleja red simbólica-emocional que define la configuración subjetiva (González, 2009).

Considerando la influencia que tiene cada contexto educativo en el sentir emocional de las personas, es una responsabilidad ética promover una educación emocional que contribuya en forma crítica a su calidad. Un principio educativo sería comprender que las emociones pueden impulsar a perjudicar o beneficiar el bienestar subjetivo de 
otras personas, pues están orientadas a dirigir la acción, por lo que es imprescindible cuidar la educación afectiva (Marina, 2016). Contribuyendo a esta percepción con un trabajo específico, de mindfulness o resiliencia (Prieto, 2018) o de relajación en la escuela (Luis-Pascual, 2014), este tipo de aprendizajes ayudarán a transformar una educación que históricamente ha priorizado metas intelectuales, en una educación que equilibra su atención en la dimensión intelectual, afectiva y motriz del ser humano.

\section{El bienestar subjetivo en la educación}

Antes de argumentar el rol del bienestar subjetivo en la educación, conviene responder las siguientes preguntas: ¿Es un deber que el estudiantado perciba un bienestar subjetivo durante su formación en los contextos educativos? La respuesta es negativa, porque en el proceso educativo el estudiantado vivenciará emociones negativas para su bienestar subjetivo, pero positivas a nivel pedagógico, ya que no todo lo que produce bienestar subjetivo es positivo para el desarrollo moral. Otra pregunta importante es: ¿Debería la educación promover el logro del bienestar subjetivo del estudiantado en el marco de la ética? La respuesta sería positiva, porque si se desea educar en valores a una persona, es necesario considerar su personalidad en forma integral. Por lo tanto, si se estableciera la meta de que el alumnado respete a sus compañeros y compañeras inmigrantes, entendiendo que toda persona extranjera en su país se encuentra sujeta a ese derecho, es importante lograr que el alumnado logre sentir emociones que favorezcan su bienestar en el acto valórico, para lo cual sería necesario desarrollar el razonamiento moral. Si durante ese mismo aprendizaje valórico, un individuo de los estudiantes sintiese emociones negativas para su bienestar, sería un indicador para la docencia de que tendría que analizar el razonamiento moral del alumnado, para identificar la posible causa de su sentir, ya que una de las metas más importante de la educación emocional sería promover un adecuado sentir emocional (Marina, 2011). En ningún caso se piensa que si ese alumno o alumna no siente bienestar subjetivo, no podrá cumplir su deber de respetar a sus pares, pero se cree que la educación puede lograr mucho más que adoctrinar al alumnado con normativas a través de un método conductista. 
Cuando se piensa el bienestar subjetivo en el marco de la ética, las emociones pierden el valor de la teoría hedonista, y toman un valor pedagógico, asociado a la finalidad educativa, de modo que con el mismo ejemplo, si un alumno siente diversión cuando se discrimina a un compañero inmigrante, esa emoción toma un valor negativo para el desarrollo del respeto.

Esta relación entre emociones positivas para el bienestar subjetivo y acciones inmorales se describe en un estudio empírico que abordó las experiencias estudiantiles de violencia en la escuela. Este identifica una relación afectiva-emocional entre las personas que componen un hecho de violencia escolar (víctima y victimario), la cual se caracteriza por las emociones percibidas en función del rol de la relación, debido a que quienes sufren la violencia experimentan emociones negativas para su bienestar subjetivo (miedo y vergüenza); mientras que quien ejerce la violencia percibe emociones positivas para su bienestar subjetivo (diversión) (Velázquez, 2005).

Incorporar el bienestar subjetivo a la educación no tiene que ser sinónimo de fomentar una moral egoísta, sino que los fines pueden ser orientados a "enlazar el mundo de las emociones con el mundo de la acción moralmente buena" (Marina, 2005, p. 37). Incluye, además, la empatía y la compasión como una propuesta de la atención plena específica tanto en la escuela como en la universidad (Viciana et al., 2018).

Considerar en el ámbito pedagógico el bienestar subjetivo en el marco de la ética, responde a un enfoque histórico cultural, basado en los derechos humanos, que exige un entorno de aprendizaje que no resulte hostil para el estudiantado, sino que, por el contrario, sea acogedor, seguro y saludable, y tenga muy presente la protección ante discriminaciones, humillaciones o agresiones, además de las diferencias sociales y sexuales, de manera que no solo atienda su aprendizaje, sino que fomente su bienestar emocional, físico y psicológico (UNICEF y UNESCO, 2008). Un ejemplo de que en Latinoamérica todavía existe un fuerte acento positivista en el currículo escolar ha sido evidenciado en un estudio realizado por el programa de las naciones unidas para el desarrollo y el fondo de las naciones unidas para la infancia, el cual analizó el aporte que tiene el currículo escolar en Chile para el bienestar subjetivo. Concluye que el enfoque potencialmente conductista del currículo, su disciplinariedad y la generación de estándares basados en esta misma, restringen la formación integral de escolares, al acotar 
su potencialidad a la dimensión cognitiva y otorgar poco espacio para abordar su subjetividad (Castillo y Contreras, 2014). No obstante, es fundamental considerar el bienestar subjetivo con un marco ético en la educación, porque, de lo contrario, se corre el riesgo de generar un adoctrinamiento afectivo de las personas, en donde el Estado, tratando de imponer sus propios criterios de felicidad, incurra en un perfeccionamiento éticamente injustificado (Briceño y Guillezeau, 2012), lo cual tiene que ser prevenido por medio de un responsable consenso en las políticas educativas, que incluya la opinión de todos los sujetos actores del proceso pedagógico.

\section{Educación moral para el desarrollo emocional}

Desarrollar correctamente las emociones, con el fin de promover los derechos humanos, se convierte en una propuesta pedagógica que resguarda las libertades individuales, para lo cual no es correcto estandarizar el valor de las emociones desde una perspectiva hedonista, pues estas no tienen un valor moral intrínseco, como se ha expresado en los anteriores ejemplos, de modo que una misma emoción puede desencadenarse en respuesta a una moral correcta o incorrecta (Steinfath, 2014). En el mismo sentido, una educación que promueve los derechos humanos comprende la formación moral de sus estudiantes, como una condición básica para el bienestar de las sociedades, tanto en el sentido existencial, como de la identidad de las personas (Barba, 2006). Esta es una formación que requiere atender la conducta, el carácter, los valores, el razonamiento y los sentimientos de las personas (Gómez, 2007). Esta propuesta de aprendizaje se desarrolla sobre la base de dilemas personales que construyen una propuesta ética que envuelve todo el proceso educativo, dependiendo de las referencias sociales y familiares que llegan a marcar la propia percepción emocional (Sánchez-Nuñez et al., 2018).

Tal como se señala, si se requiere construir, por medio de la educación, una sociedad civilizada, que supere la insensibilidad y las geografías simbólicas del mal, es menester educar las emociones desde temprana edad, pues las concepciones de justicia con equidad, solidaridad y cooperación social se relacionan con el cultivo y florecimiento de las emociones desde la infancia como parte del desarrollo humano y de nuestra formación para la humanidad, al promover una cultura política inclusiva, que ayude a las personas a comprender y sensibilizarse frente 
a su común humanidad, ya que, a través de la imaginación narrativa y empática, se podrán educar seres humanos capaces de vivir y amar en la diversidad (Quintero y Sánchez, 2016). No se trata de forzar la sensibilidad y ética del alumnado, sino de desarrollar el buen sentir, pensar y hacer, para lo cual la contemplación es un gran aporte para la conciencia y comprensión emocional. Por medio del desarrollo personal del estudiantado, se le ayudaría a no reaccionar en forma apresurada cuando el contexto lo permita y a dar una respuesta que esté gestionada por principios morales.

Las emociones, durante mucho tiempo, han sido censuradas, silenciadas, proscritas, condenadas y reprimidas en el contexto educativo (Toro, 2017); sin embargo, cada vez hay más conciencia de que pueden ser educadas, tal como señala el biólogo y epistemólogo Maturana, cuando argumenta que los seres humanos no están determinados en su origen, pues no se nace con la condición humana, sino que esta se construye en el mundo de relación con otros seres humanos. Se plantea una educación contextualizada en el entorno sociocultural, situación que otorga un carácter fundamental a la educación, entendida como un convivir en el cual se transforma el emocionar y el actuar de quienes lo hacen según las conversaciones que constituyan ese convivir (López, 2012). En consecuencia, Maturana propone un modelo bio-pedagógico, donde la finalidad de la educación sea brindar amor, pero no es el amor que es representado por la pasión de una pareja o el que se basa en deseos hacia algo o alguien, sino un amor con significado moral, entendido como la aceptación del otro ser como un legítimo otro en la convivencia, caracterizado por la bondad amorosa y la compasión. El amor ha sido considerado como la emoción básica que funda lo humano y lo social, lo cual es una condición biológica de todos los seres humanos (Ortiz, Sánchez y Sánchez, 2015).

A partir de las ideas anteriores, surge un planteamiento bioético, en donde lo humano no necesariamente se concibe desde la racionalidad occidental. Sin desconocer la importancia de la dimensión racional, se tiene que asumir que enlazada a la razón se encuentra la emoción; se justifica, así, una nueva ética de la acción humana, en donde debe existir un respeto profundo y genuino por la naturaleza y por los otros seres vivos, reconocidos como parte de una realidad de interdependencia que acerca a los seres humanos y los integra (Mazo, 2014). Este nuevo paradigma es holístico; pretende trascender el dualismo cartesiano y 
el exceso de importancia que se la ha atribuido a la razón en la constitución del ser humano, por lo que surge para reivindicar el valor de la dimensión emocional como aspecto esencial de la formación humana (Burgos, 2015). Pretende, de esta manera, establecer una verdadera educación integral, que logre superar los diferentes reduccionismos en los cuales se ve sumergida la educación formal, obsesionada con el éxito del alumnado en las diferentes pruebas estandarizadas de las asignaturas "relevantes" para el sistema educativo (énfasis intelectual), en menosprecio de las asignaturas "menos relevantes" (énfasis motriz o artístico-musical), por medio de las cuales el alumnado ve incrementada sus posibilidades de interactuar con sus pares, tomar conciencia de la corporalidad, expresar su "mundo interno" y, en general, desarrollar una adecuada afectividad.

\section{Formación emocional del profesorado}

La meta de la formación docente, antes que lograr competencias que definan al profesorado como un gran intelectual, deportista o artista, busca la formación de su personalidad, la cual no se ve favorecida con el abrumador predominio del desarrollo cognitivo en comparación con las otras dimensiones. Como se ha argumentado, la dimensión afectivo-emocional es básica, esencial y fundamental en el desarrollo personal, entendiendo que lo que define a la persona es la integración armónica de sus diversas dimensiones (Toro, 2017). Si el profesorado no es consciente de que la emotividad es moldeable, controlable y que es un medio de expresión de la personalidad (López, 2016), además de que no se encuentra determinada sino, por el contrario, puede ser educada, es muy difícil los grupos escolares lo comprendan, más aún si se piensa en los contextos pedagógicos más desfavorecidos, donde asisten niños o niñas que provienen de entornos familiares con graves problemas de violencia, drogadicción o inmoralidad, no necesariamente asociados a un tipo específico de nivel socioeconómico, ya que este tipo de problemas se observa a nivel transversal en la sociedad.

La educación emocional en la formación del profesorado se presenta como una urgente necesidad social, relegada por diferentes motivos, pero en la actualidad, existen múltiples argumentos que justifican su incorporación en el currículo preescolar, escolar y universitario. Por lo demás, no es posible que esta dimensión sea abordada 
con calidad, si no se forma en el tema al profesorado, de modo que es difícil enseñar competencias emocionales, si previamente no ha existido una adecuada formación (Escolar et al., 2017). Desde la conciencia dinámica corporal de la Escuela relajada, Luis-Pascual (2014) se propone una intervención basada en el "modo ser" frente al "modo hacer" que hace observables y conscientes las sensaciones físicas, las emociones y los pensamientos presentes en el momento de la experiencia, sin juzgarlos, desde una actitud de mayor objetividad y ecuanimidad que permite mejorar la gestión emocional, primero del propio profesorado y después de sus estudiantes. Esta necesidad de actualización se encuentra respaldada por diferentes estudios empíricos, como el realizado en la Universidad de Castilla la Mancha, en el que se consultó a estudiantes de primer y cuarto curso del grado de maestro en educación infantil y del grado de maestro en educación primaria, sobre la importancia y la carencia de formación en educación emocional para el óptimo desempeño del ejercicio profesional docente. Este concluye que todas las competencias emocionales consultadas son consideradas con una alta y muy alta importancia (Cejudo et al., 2015).

En el mismo sentido, estudios realizados en la Universidad de Playa Ancha, han identificado variados factores que atribuyen al sentimiento emocional de estudiantes de pedagogía en Educación Física en su práctica pedagógica, quienes, al ser analizados desde un aspecto temporal, suceden antes, durante y después de sus intervenciones (Mujica et al., 2015), se refieren a la importancia de la interacción docente-estudiante en el comportamiento de escolares, las metas personales, la evaluación del desempeño, la preparación de la clase y las creencias personales (Orellana et al., 2015). En España, hace más de una década, Bisquerra (2005) estableció una metodología teórico-práctica para la formación docente, con el fin de favorecer el desarrollo de competencias, como la conciencia o la regulación emocional, instituyó diferentes objetivos educativos, como el de favorecer mejores relaciones interpersonales o ejercer un autocontrol emocional en las situaciones conflictivas, los cuales representan aspectos fundamentales para el ambiente de aprendizaje y la calidad educacional. No obstante, es indispensable abordar esa propuesta con una mirada crítica, para poder dar respuesta a las dudas y planteamientos que cuestionan su inclusión en el ámbito pedagógico. Principalmente, se hará referencia al valor de las emociones, ya que como se ha mencionado, es indispensable que 
ese valor sea aplicado al campo pedagógico y no sea abordado desde perspectivas ajenas al quehacer educativo.

El enfoque de la educación emocional propuesto por Bisquerra considera que una persona educada es aquella que está en condiciones de construir su propio bienestar, colabora con el bienestar general, tiene presente que su construcción incluye la regulación de las emociones negativas y la potenciación de las positivas (Bisquerra, et al., 2016). Esta definición trae implícito un valor moral, ya que al mencionar que se orienta a la contribución del bienestar general, trasciende el riesgo de convertirse en una educación egocentrista, esto es, se orienta al bienestar como derecho social. Sin embargo, como las emociones son un indicador subjetivo del bienestar, no sería correcto que el bienestar general se construya solo por medio de la potenciación de las emociones positivas para el bienestar subjetivo, puesto que hay emociones negativas a nivel personal, pero positivas a nivel social. Además, no sería correcto en este tipo de educación que se establezcan fronteras para las emociones, clasificándolas en positivas y negativas de acuerdo con el bienestar subjetivo, ya que como se ha señalado anteriormente, una misma emoción desde un punto de vista moral, puede ser clasificada en positiva o negativa según la meta educativa. De tal forma, si se establece un objetivo educativo a nivel moral de respetar la opinión de las demás personas, y, por ejemplo, un alumno descalifica a una de sus compañeras por haber dicho una opinión contraria a la de él, y siente, posteriormente, vergüenza y se disculpa, esa emoción que para el bienestar subjetivo es negativa, para esta ocasión sería positiva, ya que en parte impulsó a rectificar el actuar.

El profesorado tiene que comprender que las emociones han sido observadas desde diferentes perspectivas académicas, pero estos valores no corresponden a la realidad educativa, por lo que es indispensable evaluarlas desde una mirada que corresponda al contexto pedagógico. Las emociones ocurren por una razón, cumplen diferentes funciones sociales, como la de preparar a una persona para defenderse ante una amenaza (supervivencia), reconocer un acto de solidaridad ante una necesidad (agradecimiento) o identificar una injusticia social (transformación social), por lo que, esencialmente, las emociones no tienen un valor implícito (bueno-malo; positivo-negativo) (Castilla del Pino, 2000), de modo que su objetivo es dirigir la atención para orientar el 
comportamiento, en función de las diferentes situaciones que han de ser enmarcadas en un contexto concreto (Reeve, 2010).

Por lo tanto, si en un contexto educativo se hace referencia a emociones negativas o positivas, se debe designar en relación con cuál aspecto asume ese valor, como por ejemplo, emoción positiva para el bienestar subjetivo. Y como se ha mencionado anteriormente, no todas las emociones positivas para el bienestar subjetivo son positivas para el logro de un objetivo pedagógico, por lo que podría ser valorada de otro modo en relación con esa meta educativa. En la práctica docente se tiene que facilitar la desidentificación de las emociones. Es decir, hacer más conscientes al estudiantado que las emociones vivenciadas no son ellos o ellas, para así tratar de poner distancia en el análisis de una emoción, para no actuar de forma reactiva (no consciente) sino con una respuesta personal (consciente).

Las emociones son multidimensionales: existen como sentimientos subjetivos, como reacciones biológicas, como agentes intencionales y como fenómenos sociales, componentes que se encuentran interrelacionados y coordinados. Específicamente, el componente intencional le da su carácter dirigido a metas para realizar las acciones necesarias a fin de afrontar las circunstancias del momento, lo cual explica el porqué las personas quieren hacer una determinada acción y por qué se benefician de sus emociones (Reeve, 2010). Las emociones motivan el cerebro para la conducta, pero la persona por medio de su razonamiento tiene que determinar si una conducta es adecuada o inadecuada socialmente, por lo que es indispensable que el profesorado sea consciente de sus emociones y evalúe la situación antes de actuar, para que su decisión no sea un acto inconsciente impulsado por su sentir, sino un acto consciente enmarcado en la ética profesional. Si se presta atención a esta valoración, se podrá conocer el significado relacional de las emociones (Lazarus, 2000) y, para una utilidad pedagógica, se debería no juzgar, evaluar o valorar la emoción en positiva o negativa. Tenemos dichas emociones (agradables, desagradables o neutras) que nos provocan apegos y aversiones; pero es la mera observación (sin intervención) la que permite hacer mucho más objetiva la respuesta, evitando que una emoción débil se pueda convertir en una emoción fuerte y que desemboque en frustración o en una conducta desproporcionada. 


\section{Consideraciones finales}

A partir de la crítica realizada al valor de las emociones, que es estandarizado a priori desde la perspectiva hedónica del bienestar, se genera la necesidad de re-pensar la educación emocional, la cual se acerca a la dirección correcta con respecto a los desafíos de la educación del siglo XXI.

El análisis realizado sobre las emociones en la educación puede resumirse en cuatro reflexiones de relevancia para una educación emocional que se encuentra en el marco de la ética.

En primer lugar, por un deber moral y siempre en el marco de los derechos fundamentales, la educación formal tiene que contribuir a que el alumnado pueda construir y experimentar, en forma adecuada, su bienestar subjetivo, entendiendo que, en esta construcción social y cultural, es muy probable que el estudiantado perciba emociones negativas para ese bienestar; no obstante, en estas circunstancias se deben utilizar metodologías que consideren la sensibilidad del alumnado y comprendan la complejidad de su emoción, para evitar aumentar el malestar subjetivo y generar un ambiente que ayude a superarlo.

En segundo lugar, la educación emocional tiene que ser considerada como una extensión de la formación moral del alumnado, ya que, de esta forma, se puede justificar el valor pedagógico de la gestión emocional en las diversas situaciones dentro del contexto educativo.

En tercer lugar, no es posible estandarizar el valor pedagógico de las emociones, pues cada situación pedagógica debe ser analizada en forma cualitativa, por su significancia moral e histórico-cultural.

Por último, se plantea que la finalidad más avanzada de la educación emocional es desarrollar buenos sentimientos emocionales y, de esta forma, promover el cumplimiento de los derechos humanos en la sociedad.

\section{Referencias}

Aguado, M., Calvo, D., Dessal, C., Riechmann, J., González, J. González, J. y Montes, C. (2012). La necesidad de repensar el bienestar humano en un mundo cambiante. Papeles de relaciones ecosociales y cambio global, 119, 49-76.

Alonso, J. (2017). Las emociones. La base neurológica del comportamiento. Barcelona: RBA. 
Barba, B. (2006). La educación moral como asunto público. Revista Iberoamericana sobre Calidad, Eficacia y Cambio en Educación, 4(1), 95-117.

Bisquerra, R. y Gemma, F. (2003). Educación emocional y medios de comunicación. Comunicar, 20, 63-68.

Bisquerra, R. (2005). La educación emocional en la formación del profesorado. Revista Interuniversitaria de Formación del Profesorado, 19(3), 95-114.

Bisquerra, R. Bisquerra, A., Cabero, M., Filella, G., García, E. López, E., Moreno, C. y Oriol, X. (2016). Educación emocional. Propuesta para educadores y familias. Bilbao: Desclée de Brouwer.

Bisquerra, R. y Hernández, S. (2017). Psicología positiva, educación emocional y el programa aulas felices. Papeles del psicólogo, 38(1), 58-65.

Bisquerra, R. y Filella, G. (2018). Análisis del vocabulario emocional en el profesorado de lengua. Revista Electrónica Interuniversitaria de Formación del Profesorado, 21(1), 161-172. doi: 10.6018/ reifop.21.1.298421

Burgos, J. (2015). Los excesos de la razón: Hacia la recuperación de las emociones en el concepto del ser humano. Franciscanum, 54(164), 97-123.

Briceño, R. y Gillezeau, P. (2012). Argumentos sobre el estado de bienestar. Negotium, 8(23), 26-66.

Cabezas, M. (2013). Juicios morales y fronteras biológicas: Más allá de la frontera razón emoción. Revista arbor, 189(762), 1-1.

Calderón, E. (2014). Universos emocionales y subjetividad. Nueva Antropología, 27(81), 11-31.

Castilla del Pino, C. (2000). Teoría de los sentimientos. Barcelona: Tusquets.

Castillo, J. y Contreras, D. (2014). El papel de la educación en la formación del bienestar subjetivo para el desarrollo humano. Una revisión al caso chileno. Santiago: UNICEF-PNUD.

Cejudo, J., López-Delgado, M., Rubio, M. y Latorre, J. (2015). La formación eneducación emocional de los docentes: Una visión de los futuros maestros. Revista Española de Orientación y Psicopedagogía, 26(3), 45-62.

Damasio, A. (2011). El error de Descartes. La emoción, la razón y el cerebro humano. Barcelona: Destino. 
Escolar, M. (2017). Educación emocional en los profesores de educación infantil: Aspecto clave en el desempeño docente. Contextos Educativos, 20, 113-125.

Galí,A. F. (2018). Defusión, minfulness y valores personales (Tesis doctoral). Universidad Autónoma de Barcelona, Barcelona, España.

García, M. (2002). El bienestar subjetivo. Escritos de Psicología, 6, 18-39.

Gómez, G. (2007). La educación moral hoy. Theoria, 16(1), 5-6.

González, F. (2009). La significación de Vygotski para la consideración de lo afectivo en la educación: Las bases para la cuestión de la subjetividad. Revista Electrónica Actualidades Investigativas en Educación, 9, 1-24.

Guedes, S. y Álvaro, J. (2010). Naturaleza y cultura en el estudio de las emociones. Revista Española de Sociología, 13, 31-47.

Holodynski, M. y Friedlmeier, W. (2006). Development of emotions and emotion regulation. Nueva York: Springer.

Lazarus, R. (2000). Estrés y emoción. Manejo e implicaciones en nuestra salud. Bilbao: Desclée de Brouwer.

López, B. (2016). Aprendizaje emocionante. Neurociencia para el aula. Madrid: Biblioteca de Innovación Educativa.

López, M. (2012). Emociones, lenguaje, amor y vida: Un compromiso con la acción. Revista Interuniversitaria de Formación del Profesorado, 74(26.2), 189-203.

López-Ros, V., Pradas, R. y Font, R. (2014). Educación Física en primaria. Desarrollo curricular y estrategias para la práctica docente. Barcelona: Universitat de Barcelona.

Luis-Pascual, J. (2014). Escuela relajada, mindfulness y educación. En J.L. Bizelli y C.B. Gomide de Souza (Eds.), Faces da escola em Ibero-América (pp. 165-180). Araraquara: FCL.

Marina, J. (2005). Precisiones sobre la educación emocional. Revista Interuniversitaria del profesorado, 54, 27-44.

Marina, J. (2011). El cerebro infantil: La gran oportunidad. Barcelona: Planeta.

Marina, J. (2016). Vence tus miedos. Cuaderno de actividades. Barcelona: Ariel.

Maturana, H. (2001). Emociones y lenguaje en educación y política. Santiago: Dolmen. 
Mazo, W. (2014). Elementos constitutivos para una bioética en Humberto Maturana. Revista Lasallista de Investigación, 11(1), 181-191.

Myers, D. y Diener, E. (1995). Who is Happy? Psychological Science, $6(1), 10-19$.

Montero, J. (2016). ¿Pueden los derechos naturales hacer alguna contribución a la filosofía de los derechos humanos? CRÍTICA. Revista Hispanoamericana de Filosofía, 48(144), 61-88.

Mora, F. (2017). Neuroeducación. Solo se puede aprender aquello que se ama. Madrid: Alianza.

Mujica, F., Orellana, N. y Marchant, F. (2015). Atribución emocional en la formación inicial del profesorado de educación física: La práctica educativa. Revista Motricidad Humana, 16(2), 54-60.

Orellana, N., Mujica, F. y Luis-Pascual, J. C. (2015). Atribución causal de las emociones en la formación inicial del docente de educación física. Revista Iberoamericana de Estudos em Educação, 10(4), 1250-1260.

Ortiz, A., Sánchez, J. y Sánchez, I. (2015). Los modelos pedagógicos desde una dimensión psicológica espiritual. Revista Científica General José María Córdova, 13(15), 183-194.

Perales, F. (2000). Didáctica de las ciencias experimentales. En L. Rico y D. Madrid (Eds.), Fundamentos didácticos de las áreas curriculares (pp. 17-64). Madrid: Síntesis.

Platts, M. (2010). ¿Quiénes tienen derechos humanos? Isonomia. Revista de Teoría y Filosofia del Derecho, 33, 125-145.

Prieto, M. (2018). La psicologización de la educación: Implicaciones pedagógicas de la inteligencia emocional y la psicología positiva. Educación, 21(1), 303-320. doi: 10.5944/educXX1.16058

Quintero, M. y Sánchez, K. (2016). Emociones morales y políticas en elparadigma del mal: El (no) lugar de la infancia. Investigación \& Desarrollo, 24(2), 240-266.

Rebollo, M., Hornillo, I. y García, R. (2006). El estudio educativo de las emociones: Una aproximación sociocultural. Teoría de la Educación. Educación y Cultura en la Sociedad de la Información, 7(2), 28-44.

Rebollo, M. y Hornillo, I. (2010). Perspectiva emocional en la construcción de la identidad en contextos educativos: Discursos y conflictos emocionales. Revista de Educación, 353, 235-263.

Redorta, J. y Obiols, M. (2006). Emoción y conflicto. Paidós: Barcelona. 
Reeve, J. (2010). Motivación y emoción. Santa Fe: McGraw-Hill.

Rodríguez-Fernández, A. y Goñi-Grandmontagne, A. (2011). La estructura tridimensional del bienestar subjetivo. Anales de Psicología, 27(2), 327-332.

Ruiz, V. (2007). Derechos humanos, universales. En-claves del pensamiento, 1(1), 155-166.

Salmurri, F. (2015). Razón y emoción. Recursos para aprender y enseñar a pensar. Barcelona: RBA.

Sánchez-Nuñez, M. T., Medina, C. R. y Rubio, N. G. (2018). Gender Differences in Perceptive Emotional Adjustment of Parents on Their Children's Emotional Intelligence. Psychology, 9, 124143. doi: 10.4236/psych.2018.91009

Sarramona, J. (2008). Teoría de la educación. Barcelona: Ariel.

Steinfath, H. (2014). Emociones, valores y moral. Universitas Philosophica, 31(63), 71-96.

Soriano, E. y Cala, V. (2018). School and emotional well-being: A transcultural analysis on youth in Southern Spain. Health Education, 118(2), 171-181.

Toro, J. (2017). Educar con co-razón. Bilbao: Desclée de Brouwer.

Unanue, W., Martínez, D., López, M. y Zamora, L. (2017). El rol del bienestar subjetivo para medir el progreso de las naciones y orientar las políticas públicas. Papeles del psicólogo, 38(1), 26-33.

UNICEF y UNESCO. (2008). Un enfoque de la educación para todos basado en los derechos humanos. New York: Fondo de las Naciones Unidas para la Infancia.

Uribe, I., Gallo, L. y Fernández, A. (2017). Trazos de una educación hedonista. Movimento, 23(1), 339-349.

Valdés, V. y Gutiérrez, P. (2018). Las urgencias pedagógicas en la sociedad del aprendizaje y el conocimiento. Un estudio para la reflexión sobre la calidad en el nuevo modelo educativo. Multidisciplinary Journal of Educational Research, 8, 1-28. doi: 10.17583/remie.2018.3199

Vásquez, C. (2009). El bienestar de las naciones. En C. Vásquez y G. Hervás (Eds.), La ciencia del bienestar: Fundamentos de una Psicología Positiva (pp. 103-141). Madrid: Alianza.

Vásquez, C., Hervás, G., Rahona, J. y Gómez, D. (2009). Bienestar psicológico y salud: Aportaciones desde la psicología positiva. Anuario de la Psicología Clínica y de la Salud, 5, 15-28. 
Velázquez, L. (2005). Experiencias estudiantiles con la violencia en la escuela. Revista Mexicana de Investigación Educativa, 10(26), 739-764.

Viciana, V., Fernández Revelles, A. B., Linares, M., Espejo, T., Puertas, P. y Chacón, R. (2018). Los estudios universitarios y el mindfulness. Una revisión sistemática. REICE. Revista Iberoamericana sobre Calidad, Eficacia y Cambio en Educación, 16(1), 119-135. doi: 10.15366/reice2018.16.1.008

Zaccagnini, J. (2008). La comprensión de la emoción. Una perspectiva psicológica. En M. Jiménez (Ed.), Educación emocional y convivencia en el aula (pp. 31-84). Madrid: Ministerio de Educación, Política Social y Deporte.

Zubieta, E., Muratori, M. y Fernández, O. (2012). Bienestar subjetivo y psicosocial: Explorando diferencias de género. Salud \& Sociedad, 3(1), 66-76. 